\title{
Particular applications of infrared thermography temperature measurements for diagnostics of overhead heat pipelines
}

by T. Kruczek*

*Institute of Thermal Technology, Silesian University of Technology, Konarskiego 22, 44-100 Gliwice, Poland

\section{Abstract}

Different parameters influence the results of infrared measurements of temperature. The emissivity of the surface under consideration as well as the temperature of the ambient elements may be specified among these parameters. Convective and radiative heat transfer takes place between thermal pipelines and its surrounding. In the case of low temperature of the sky, the radiation heat exchange is very intensive and the temperature of the top part of the horizontal pipeline is much lower than the bottom part. This phenomenon has been confirmed by calculations carried out by means of CFD Fluent. The aforementioned phenomenon causes difficulties during evaluation of the quality of the thermal insulation of the pipeline. Circumstances connected with this phenomenon have been recognised and explained in this paper. Examples of calculations have been made and the results have been presented.

Denotations and subscripts

\begin{tabular}{|l|l|l|l|l|l|l|}
\hline \multicolumn{2}{|c|}{ Denotations } & \multicolumn{2}{|c|}{ Subscripts } \\
\hline $\boldsymbol{T}$ & - & temperature, K & $\boldsymbol{A t}$ & - & atmospheric air \\
\hline $\boldsymbol{\varepsilon}$ & - & emissivity & $\boldsymbol{G}$ & - & ground \\
\hline $\boldsymbol{\Delta}$ & - & $\begin{array}{l}\text { difference of } \\
\text { temperature, K }\end{array}$ & $\boldsymbol{S}$ & - & $\begin{array}{l}\text { external shell of } \\
\text { pipeline }\end{array}$ \\
\hline & & & $\boldsymbol{S k}$ & - & sky \\
\hline
\end{tabular}

1. Introduction

Thermovision examination of thermal insulation of the pipelines is a very effective and modern method. The result of the temperature thermovision measurement is influenced by many parameters, especially by emissivity of the examined surface and the temperature of the surrounding elements. Convective and radiative heat transfer takes place between thermal pipelines and its surrounding [11]. Generally, the surroundings of the external pipeline consists of two surfaces: hypothetical sky surface and ground surface [7]. Normally, the temperatures of these elements are different. In the case of low temperature of the sky, due to very intensive radiative emission of the heat, the temperature of some parts of the pipeline shell may drop below the temperature of the atmosphere [8]. Examples of such phenomena can be seen in Fig. 1. Temperature values in these points are printed near the crosses which can be found on the external surface of the pipelines (Fig. 1). It can be noticed that the temperature of the shell on the upper part of the pipelines is lower than the temperature of atmospheric air.

\section{Application of CFD fluent software for the analysis of heat exchange between the pipeline and the surroundings}

The packages of CFD (Computational Fluid Dynamics) make it possible to carry out numerical analysis or simulation of processes connected with fluid flow, heat flow processes, chemical reactions and other processes [1]. This is achieved by the numerical solution of equations describing these phenomena. The package Fluent version 6.2.16 was used to analyse the heat exchange processes taking place on the external surface of the overhead pipeline shell.

In the application procedure of Fluent calculations the following steps may be specified: formulation of a geometrical model, numerical net creation, pre-processing, solving the problem, postprocessing. During the creation of the geometrical model, the following components have been distinguished: hypothetical isothermal surface of the sky (or real laboratory roof), isothermal surface of the ground (laboratory bottom), a tube with hot fluid inside, thermal insulation, shell covering the thermal insulation.

\section{Comparison of numerical calculation and laboratory measurement results}

For the research of the heat exchange process on outer surface of pipeline shell the measurements in the laboratory have been carried out. During these experiments the following temperatures were measured by means of thermocouples: the wall temperature of electrically heated pipeline tube, the temperature of external shell of insulation and the output of electrical heater. Unfortunately, at this moment there was no possibility of carrying out the experiments in natural environment.

Additionally, for the technical parameters and geometry of the laboratory stand, the numerical calculations by means of Fluent package have been made as well. The technical characteristic of this experimental facility is 
presented in Table 1. Next, the measurement and calculation results have been compared. The results of the comparison are presented in Fig. 2. The arrow on the right hand side shows the start point and direction of collecting the data around the pipeline shell. The experimental results did satisfactorily overlap with the calculation results.

\section{Results of numerical calculations for real pipeline}

For these calculations the geometrical model 2D has been applied. The horizontal pipeline was located in the middle of the space of $10 \mathrm{~m}$ in height and $50 \mathrm{~m}$ in width. Air with uniform and determined velocity flows into this space. As a result of calculations, velocity field and air temperature field, as well as the shell temperature distribution around the pipeline have been obtained. In order to examine the problem, numerical calculations have been carried out for the data gathered in Table 1. Only the value of one parameter was being changed during multiple calculations, whereas the values of the remaining parameters were fixed. The calculation results are presented in Figs $3 \div 7$.

Table 1. Technical parameters of laboratory stand and input data for numerical calculations

\begin{tabular}{|l|c|c|c|}
\hline \multicolumn{1}{|c|}{ Specification } & Dimension & $\begin{array}{c}\text { Laboratory } \\
\text { stand }\end{array}$ & Pipeline \\
\hline Temperature of the tube, Figs 2a/2b & $\mathrm{K}$ & $478 / 544$ & 473 \\
\hline Temperature of atmospheric air & $\mathrm{K}$ & 293 & 276 \\
\hline Temperature of the roof/sky & $\mathrm{K}$ & 292 & 243 \\
\hline Bottom/ground temperature & $\mathrm{K}$ & 292 & 273 \\
\hline Wind velocity & $\mathrm{m} / \mathrm{s}$ & - & 1.0 \\
\hline Outer diameter of the tube without insulation & $\mathrm{m}$ & 0.273 & 0.273 \\
\hline Outer diameter of the tube with thermal insulation & $\mathrm{m}$ & 0.473 & 0.473 \\
\hline Average coefficient of insulation thermal conductivity & $\mathrm{W} / \mathrm{m} \mathrm{K}$ & 0.067 & 0.046 \\
\hline Emissivity of the shell surface & - & 0.05 & 0.90 \\
\hline
\end{tabular}

Figure 3 presents the distributions of the shell temperature around the pipeline. Fig. 3a shows temperature distribution for emissivity $\boldsymbol{\varepsilon}=0.0$. It means that radiative emission does not exist on the shell surface and only convective heat transfer takes place. The shell temperature around the pipeline is diversified but horizontally symmetric; the maximal difference amounts to about $6.5 \mathrm{~K}$. Figs 3b, 3c deal with shell emissivity of 0.05 and 0.2 . These values are characteristic of new metal shells although such pipelines are usually not examined. The typical shell emissivity of the pipeline with old shell amounts to about 0.9; see Fig. 3d. Strong diversification of the shell temperature between the upper and bottom part of the pipeline can be observed in this case. In order to calculate properly the heat losses in this case or evaluate the quality of thermal insulation, this phenomenon should be taken into consideration.

Figure 4 presents the influence of sky temperature on the distributions of the protection shell temperature around the pipeline. Low temperature of the sky causes considerable temperature differences, denoted by $\Delta \boldsymbol{T}_{S}$, between upper and bottom part of the pipeline shell. Besides, in the case of low sky temperature, the temperature of the upper part of the pipeline shell drops below the atmospheric air temperature; see Fig. 4a. Moreover, the shape of the temperature profile of the shell also changes along with the sky temperature. These facts cause some problems with the right analysis of the measurement data.

Figure 5 shows calculation results for different values of shell emissivity. It may be noticed that for shell emissivity bigger than 0.5 there are regions on the shell surface with the temperature lower than the temperature of atmospheric air. This diagram has been elaborated on the basis of results of multiple calculations with diversified shell emissivity.

It can be concluded from the diagram in Fig. 6 that the difference between the minimal temperature (temperature of the upper part of the pipeline) and the maximal shell temperature amounts to about $8 \mathrm{~K}$ for the considered pipeline and calculation conditions. This difference practically does not depend on the temperature of atmospheric air.

On the basis of Fig. 7 we may analyse the influence of the sky temperature on the average shell temperature and its diversification on the shell surface. Generally, an increase in the sky temperature causes a decrease in shell temperature differences. Thus, the conditions for the thermovision measurements are improved and further analysis of measurement data is easier.

\section{Comparison of CFD calculations with other methods}

Calculation results obtained by means of Fluent package have been compared with the results achieved by known methods recommended in literature [4, 6], local standards [2, 3] or in European standards [9]. Figs 8 and 9 show calculation results for input data presented in Table 1. The solid line presents distribution of the protective shell temperature around the pipeline. This temperature distribution has been calculated by means of Fluent software. Additionally, horizontal straight lines are gathered to present temperature values of the shell obtained with the use of other methods [2, 3, 4, 6, 9]. These values have been determined on the basis of the 
condition that the heat flux conducted through the thermal insulation is equal to the heat flux emitted from the external surface of the protective shell into the environment.

The succession of the lines in the diagram is the same as in the legend. In this figure it can be seen that the average temperature of the shell calculated on the basis of Fluent is almost equal to the temperature of atmospheric air. Moreover, the temperature of the shell calculated by means of other methods [2, 3, 4, 6, 9] is higher by $5 \div 7 \mathrm{~K}$ than the average temperature obtained from Fluent calculations. Simultaneously, the shell temperature between position $0 \div 0.4 \mathrm{~m}$ and $1.1 \div 1.5 \mathrm{~m}$ (Fig. 8) is lower than the temperature of atmospheric air. This situation is characteristic of high values of shell emissivity (in this case $\boldsymbol{\varepsilon}=0.9$ ), which is typical of old and rusty protective shells.

For low values of shell emissivity, the temperature of the shell is higher than the atmospheric air temperature (for the whole circumference of the pipeline). Besides, the average shell temperature calculated by means of the Fluent package almost overlaps with the temperature calculated with the use of the method recommended in [9], especially for emissivity equal to about 0.05 ; see Fig. 9. It means that the equations for the calculations of heat losses given in standard [9] may be used for the determination of heat losses only for new pipelines with small emissivity of the protective shell. Therefore, they are not directly suitable for the calculation of heat losses in the case of old and rusty sheet of the shell.

\section{Final remarks}

Application of the infrared thermography technique for determination of temperature distribution on the external shell insulation of the overhead heat pipelines is a very effective measurement technology. Knowledge of the temperature of the pipeline shell makes it possible to calculate locally the heat losses from the pipeline $[3,4,5,6,9,10]$. Determination of the heat losses makes it possible to evaluate the quality and technical state of thermal insulation of the pipeline.

Temperature distribution of the external shell of the overhead pipeline is significantly influenced by relatively low temperature of the sky. In this situation the temperature of the upper part shell may drop below the temperature of atmospheric air. This phenomenon has been confirmed by calculations carried out by means of CFD Fluent. The aforementioned phenomenon causes difficulties during evaluation of the quality of the thermal insulation of the pipeline and determination of the pipeline heat losses.

In such a situation the infrared thermography measurement results are still useful but during the calculation of heat losses from the pipelines this phenomenon has to be taken under consideration. Some results of numerical calculation have been verified by means of laboratory measurements.

It has been noticed on the basis of numerical calculations that the difference between the minimal temperature of the pipeline shell (temperature of the upper part) and the maximal shell temperature is approximately constant and practically does not depend on the temperature of atmosphere; Fig. 5. This observation is very useful during the estimation of the medium shell temperature responsible for heat losses of the pipeline.

The mechanisms of the influence of various parameters were recognised and analysed in this work. It is possible to take into account all measurement circumstances and make necessary corrections of measurement results in order to draw proper conclusions concerning the quality of pipeline thermal insulation. The author has examined many different pipelines at various conditions. However, an easier solution is to select the measurement period with appropriate meteorological conditions. It is also advisable to carry the measurements out on days with small wind velocity and with not too low sky temperature. This conditions are characteristic of weather with humid atmospheric air and cloudy sky.

\section{REFERENCES}

[1] CFD Fluent. User's Guide Fluent 6.2 Documentation. Fluent Inc. January 2005.

[2] Finnish Standards SFS 3977. Insulation of pipes, vessels and equipment, dimensioning, 1999.

[3] German Standard, VDI 2055. Thermal Insulation of Heated end Refrigerated Industrial and Domestic Installations. Calculations, Guarantees, Measuring and Testing. Methods, Quality Assurance, Supply Conditions, 1994.

[4] Górecki J.: Heat distribution networks (in Polish). Oficyna Wyd. Pol. Wrocławskiej, Wrocław 1997.

[5] Górzyński J.: Industrial thermal insulations (in Polish). Wyd. Sorus, Poznań 1996.

[6] Kostowski E.: Heat transfer (in Polish). Wyd. Politechniki Śląskiej, Gliwice 2000.

[7] Kruczek T.: Analysis of the influence of external conditions on thermovision measurement results (in Polish). Proc. of Conference „V Krajowa Konferencja - Termografia i Termometria w Podczerwieni”, Ustroń, 2002, s.327-332.

[8] Kruczek T., Węcel G., Bulanda A.: Analysis of the thermovision measurements results of the heat-pipelines with the application of the CFD Fluent package (in Polish). Proc. of Conference „VII Krajowa Konferencja Termografia i Termometria w Podczerwieni", Ustroń, 2006, s. 125-129.

[9] Polish Standard PN-EN-ISO 12241. Thermal insulation for building equipment and industrial installations (in Polish), 2001 (translation, ISO 12241, 1998).

[10] Polish Standard PN-B-02421. Thermal insulation of pipelines, accessories and devices (in Polish). 2000.

[11] Siegel R., Howell J. R.: Thermal Radiation Heat Transfer, Ill-rd edition, Hemisphere Publishing Corporation, Washington, 1992. 


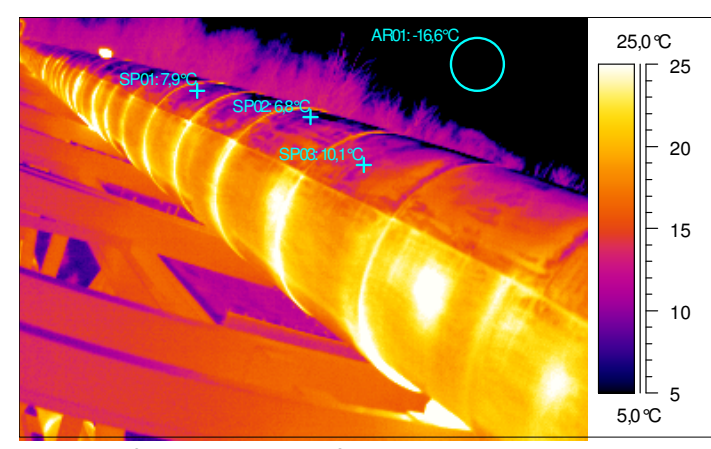

$T_{A t}=13^{\circ} \mathrm{C}, T_{S k}=-16.6^{\circ} \mathrm{C}$

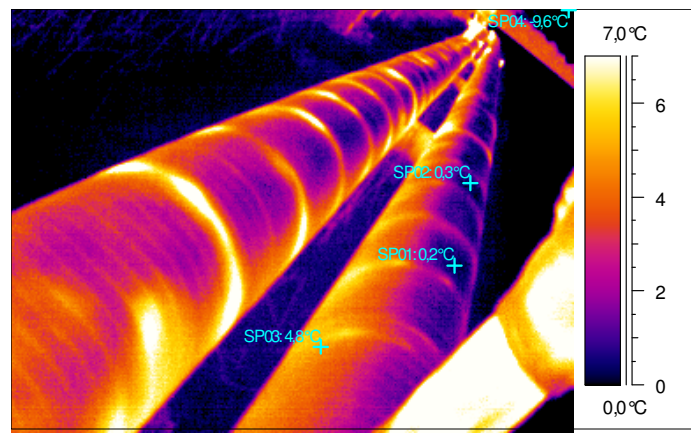

$\boldsymbol{T}_{\boldsymbol{A} t}=4.5^{\circ} \mathrm{C}, \boldsymbol{T}_{S k}=-9.6^{\circ} \mathrm{C}$

Fig. 1. Example results of thermovision examination of heat pipelines
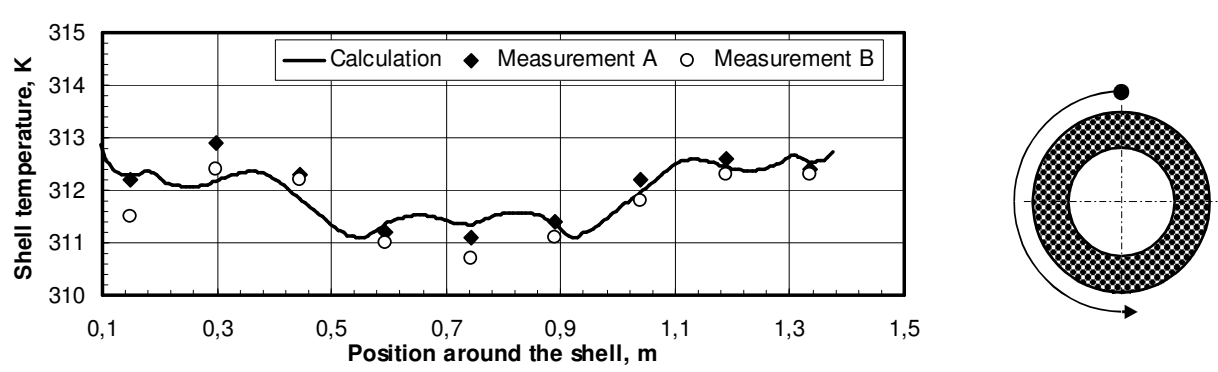

Fig. 2a. Temperature of pipeline shell - measurement and calculation results, heat flux $80 \mathrm{~W} / \mathrm{m}^{2}$
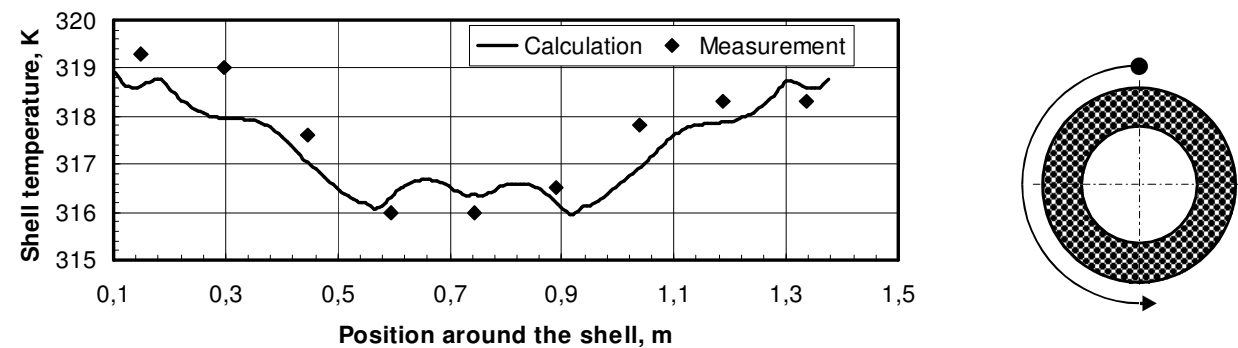

Fig. 2b. Temperature of pipeline shell - measurement and calculation results, heat flux $120 \mathrm{~W} / \mathrm{m}^{2}$

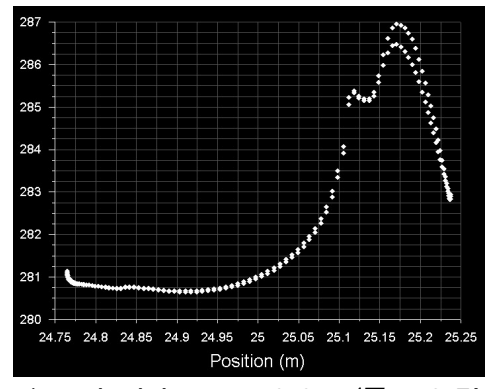

a) emissivity $\varepsilon_{S}=0.0, \Delta T_{S}=6.5 \mathrm{~K}$

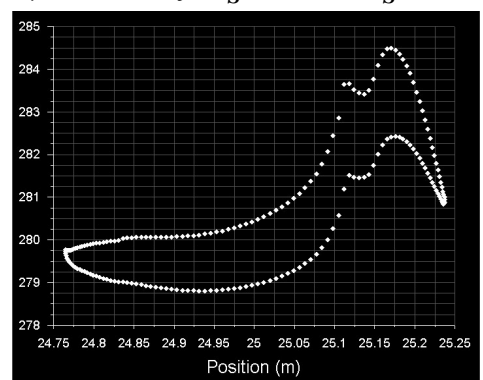

c) emissivity $\varepsilon_{S}=0.2, \Delta T_{S}=5.8 \mathrm{~K}$

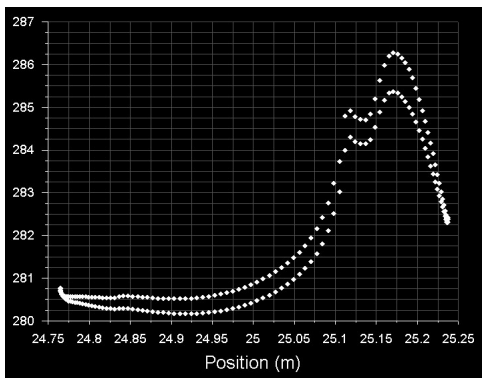

b) emissivity $\varepsilon_{S}=0.05, \Delta T_{S}=6 \mathrm{~K}$

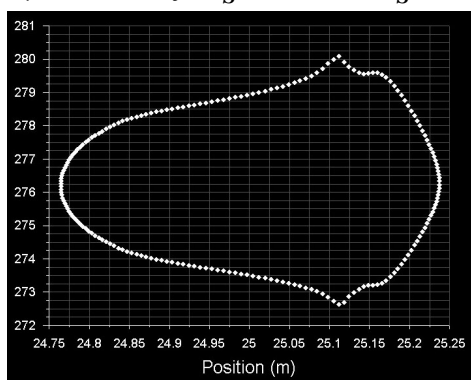

d) emissivity $\boldsymbol{\varepsilon}_{S}=0.9, \Delta \boldsymbol{T}_{S}=7.5 \mathrm{~K}$

Fig. 3. Distributions of the temperature of the shell around the pipeline for various values of emissivity of the shell; horizontal axis - position along wind way (position $=25 \mathrm{~m}$ - vertical symmetry axis of the pipeline), vertical axis of diagram - temperature of the pipeline shell 


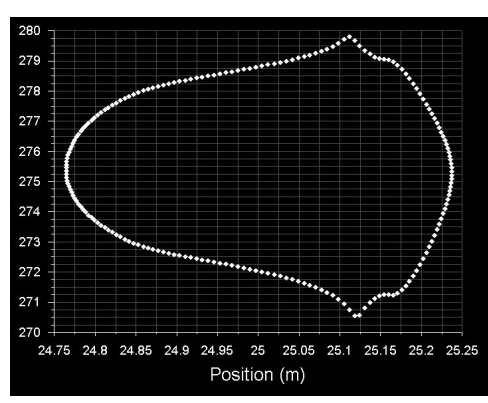

a) $T_{S k}=233 \mathrm{~K}, \Delta T_{S}=9.3 \mathrm{~K}$

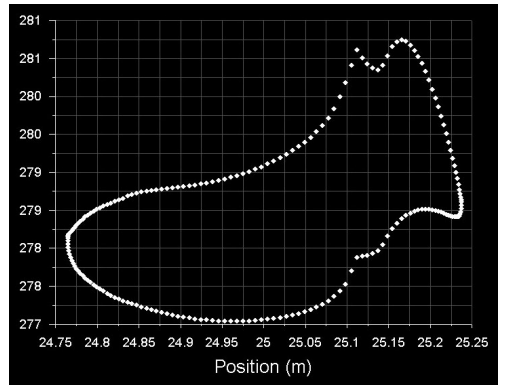

b) $\boldsymbol{T}_{S k}=263 \mathrm{~K}, \Delta \boldsymbol{T}_{S}=4.2 \mathrm{~K}$

Fig. 4. Distributions of the temperature of the shell around the pipeline for various temperatures of the sky $\boldsymbol{T}_{\boldsymbol{S} k}$, horizontal axis - position along wind way (position $=25 \mathrm{~m}-$ vertical symmetry axis of the pipeline), vertical axis - temperature of the pipeline shell

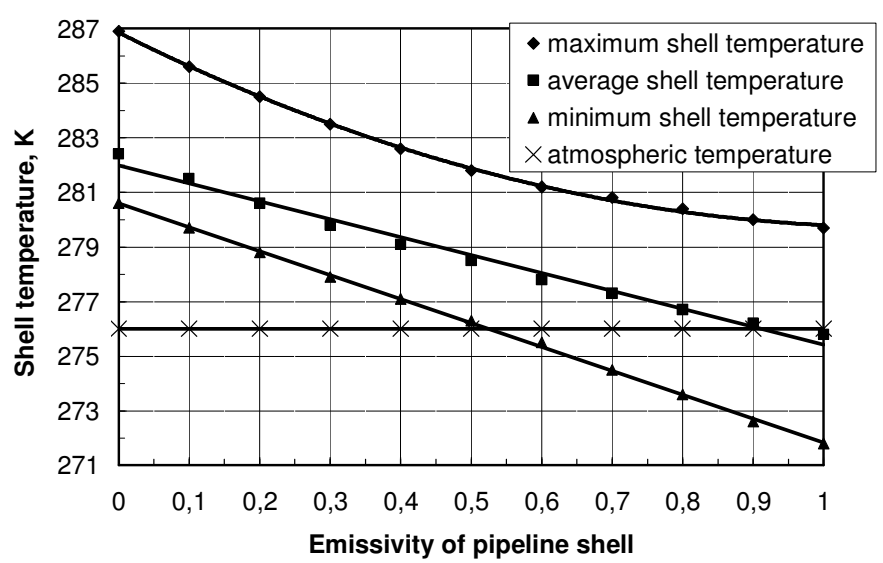

Fig. 5. Influence of the shell emissivity on the temperature of pipeline shell

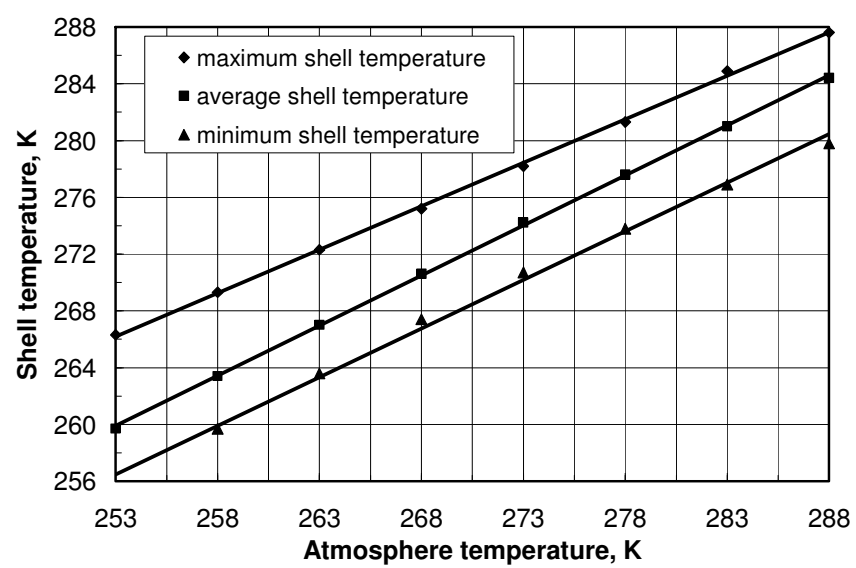

Fig. 6. Influence of the atmospheric air temperature on the temperature of the pipeline shell 


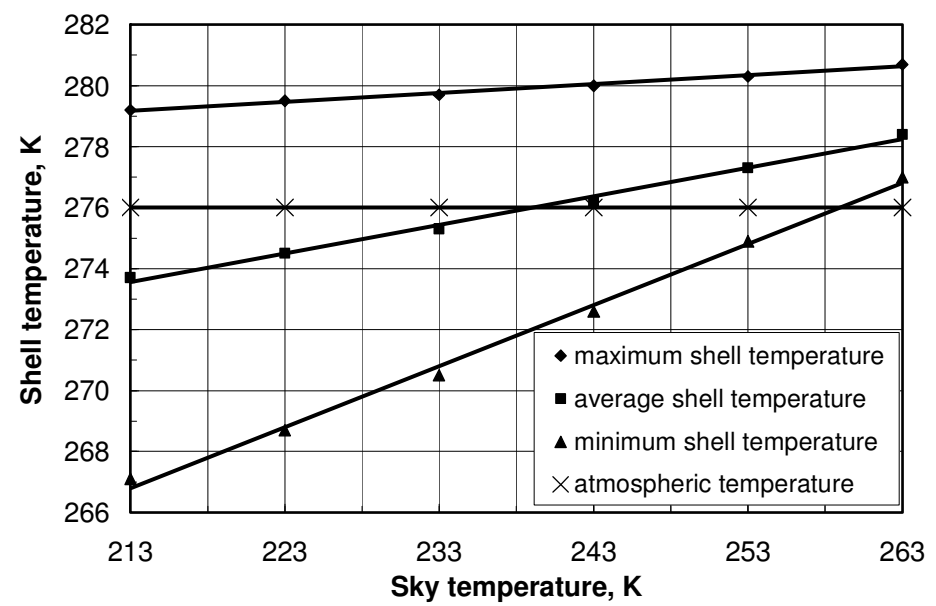

Fig. 7. Influence of the sky temperature on the temperature of the pipeline shell
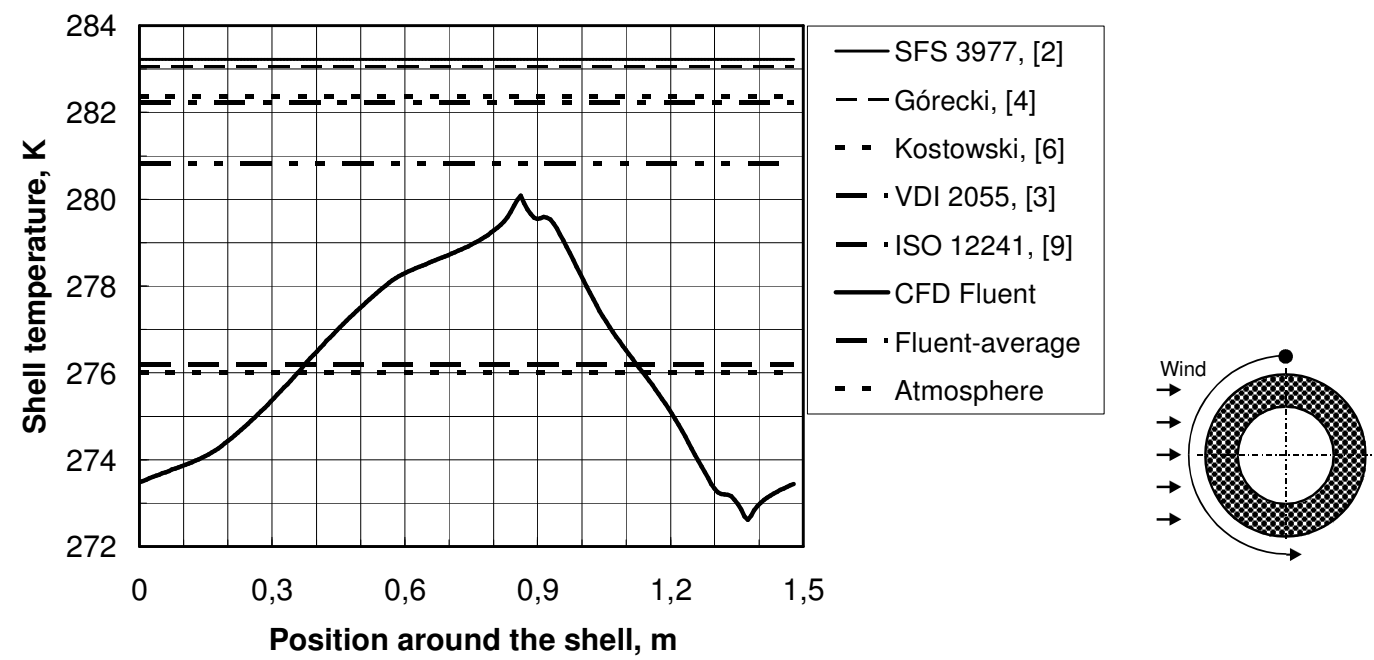

Fig. 8. Distributions of the temperature around the pipeline shell obtained by means of different methods, emissivity of the shell is equal to 0.9; on the right - start point and direction of results collection around the pipeline shell

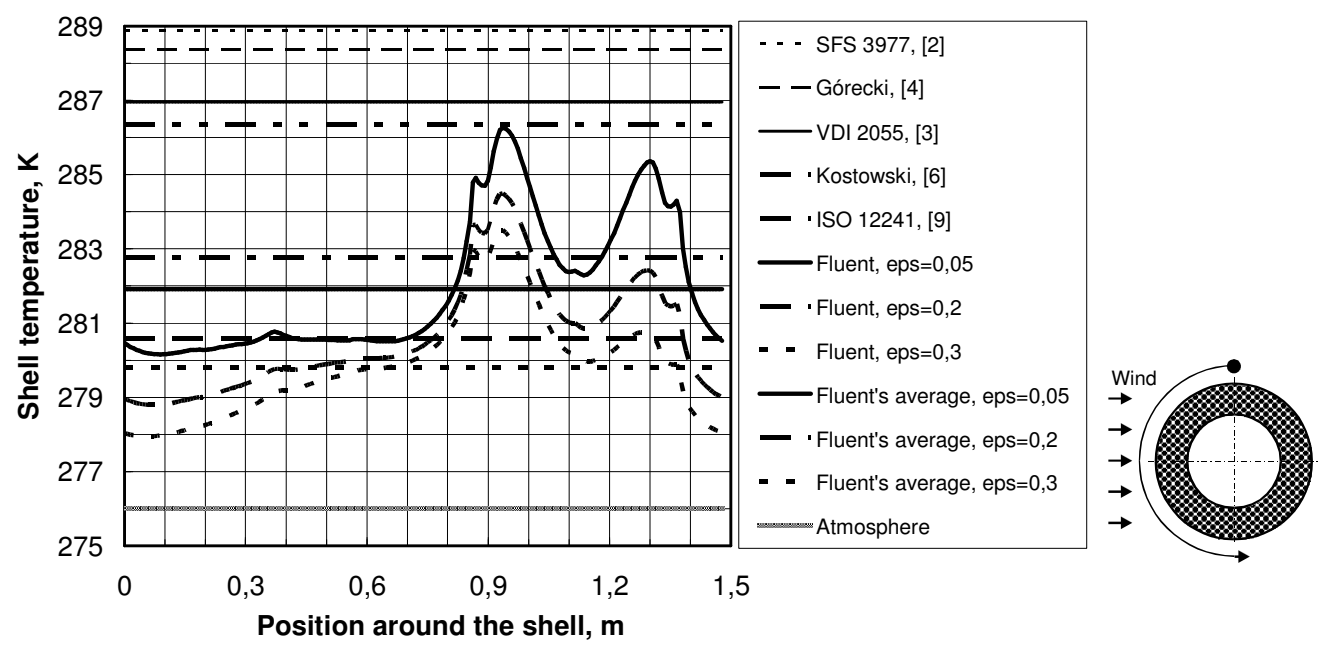

Fig. 9. Distributions of temperature around the pipeline shell obtained by means of different methods, emissivity of shell is equal to $0.05,0.2,0.3$ as specified in the legend; on the right - start point and direction of results collection around the pipeline shell 